\title{
Tunable Electrical Conductivity of Flexible Metal-Organic Frameworks
}

\author{
Sanggyu Chong ${ }^{1 \dagger}$, Sven M. J. Rogge ${ }^{2 \dagger}$, Jihan Kim ${ }^{\text {* }}$
}

1: Department of Chemical and Biomolecular Engineering, Korea Advanced Institute of Science and Technology (KAIST), Daejeon 34141, South Korea

2: Center for Molecular Modeling (CMM), Ghent University, Zwijnaarde 9052, Belgium

$\dagger$ These authors contributed equally to this work.

* Author to whom correspondence should be addressed: jihankim@kaist.ac.kr 
ABSTRACT: We present the computational design of naphthalene diimide (NDI)-containing metal pyrazolate MOFs (M(NDIDP), M = Zn, Co, Fe) judiciously bestowed with both framework flexibility and electrical conductivity. M(NDIDP) MOFs exhibit "wine rack" type flexibility, allowing them to partake in pressure-induced structural transitions at low pressures and room temperature. The MOFs are also equipped with closely packed redox-active NDI moieties, which results in dispersive conduction band minima for n-type charge transport and conductivity. Remarkably, structural transitions of the designed MOFs are found to facilitate the rearrangement of NDI moieties. As a result, conductivity of M(NDIDP) MOFs can be finely tuned, which is evidenced by the gradual shift from $1 \mathrm{D}$ to $2 \mathrm{D}$ charge transport and up to 7.5fold reduction in carrier effective mass $\left(2.76 m_{0}\right.$ to $\left.0.37 m_{0}\right)$ when transitioning between different structural configurations. The unprecedented discovery of flexible MOFs with tunable electrical conductivity presented in this work firmly establishes MOFs as versatile candidate materials for multifunctional electronics.

KEYWORDS: metal-organic framework, electrical conductivity, flexibility, stimuliresponsive behavior, density functional theory 


\section{Introduction}

The high modularity of metal-organic frameworks (MOFs) with multiple design parameters (e.g., metals ions/clusters, ${ }^{1}$ organic linkers, ${ }^{2}$ topologies, ${ }^{3}$ and $\operatorname{defects}^{4,5}$ ) has led to the discovery of a large number of MOFs with a wide range of properties and functionalities. Judicious choice of the framework components makes it possible to tailor these materials for a variety of applications, ranging from gas storage and separation ${ }^{6}$ to catalysis, ${ }^{7}$ drug delivery, ${ }^{8}$ water harvesting, ${ }^{9,10}$ etc. More recently, research has shown that electrical conductivity can be induced in MOFs. ${ }^{11-15}$ Due to their porosity and conventional use of electrically inactive components (i.e., closed-shell metals, $\sigma$-bridged organic linkers), MOFs have long been considered as electrical insulators. However, by synthesizing MOFs from redox-active metals and linkers that allow significant delocalization of charge carriers, it becomes possible to secure long-range charge transport (CT) pathways in MOFs to induce conductivity. Experimental conductivities of MOFs have reached up to $2500 \mathrm{~S} / \mathrm{cm},{ }^{16-18}$ and as such, they have been successfully integrated in field-effect transistors, ${ }^{19}$ supercapacitors,${ }^{20}$ chemiresistive sensors, ${ }^{21-}$ ${ }^{23}$ and electrocatalysts. ${ }^{24}$ While substantial progress has been made, research thus far has mainly focused on inducing the conductivity in MOFs. To make further breakthroughs, however, focus should be shifted towards synergistically combining conductivity with other properties unique to MOFs, allowing novel application schemes unfeasible in other materials.

One of the most intriguing properties of MOFs that differentiates them from other materials is framework flexibility. ${ }^{25}$ Generally, MOFs are considered to be more flexible than other solid crystalline materials as they inherently possess several structural degrees of freedom (e.g., linker rotation, lattice deformation). Here, however, we specifically refer to flexibility as those cases where MOF constituents participate in large amplitude structural transitions whilst conserving the original framework topology. Such flexible MOFs are capable of undergoing significant expansion or contraction of the framework, which can drastically alter their pore geometry and internal arrangement of framework components. This can be viewed akin to a switch or a dial, as the framework is able to effectively transition between clearly distinct states or structural configurations via external stimuli (e.g., guest adsorption, temperature, light, pressure $).{ }^{26,27}$ As such, framework flexibility can effectively instill new external parameters (e.g., stimuli and time) for the control of MOF properties, opening up many new applications. ${ }^{28}$ Previous studies have utilized the flexibility of MOFs for selective chemical sensing, ${ }^{29}$ switchable catalysis, ${ }^{30}$ or band gap modulation. ${ }^{31}$ However, to the best of our knowledge, no 
one has linked framework flexibility directly with the electrical conductivity of MOFs, which could potentially lead to tunable electrical conduction properties.

In this computational study, we have performed the modular design of MOFs that simultaneously harness both framework flexibility and electrical conductivity. To guarantee the framework flexibility of our designed MOFs, a well-known flexible MOF (i.e., M(BDP), ${ }^{32-34}$ $\mathrm{H}_{2} \mathrm{BDP}=1$,4-di(1H-pyrazol-4-yl)benzene) was adopted as the template framework. Then, central benzene groups of the linkers were replaced with redox-active 1,4,5,8-naphthalene diimide (NDI) moieties to impart electrical conductivity, resulting in the newly proposed MOFs referred to as M(NDIDP). Density functional theory (DFT) modeling of M(NDIDP) MOFs was performed to discover the presence of multiple metastable phases with vastly different internal arrangement of the NDI moieties. Next, molecular dynamics (MD) simulations with DFTderived force fields were carried out to show that the MOFs can undergo pressure-induced phase transitions at room temperature, firmly establishing their framework flexibility. DFT electronic band structure analysis revealed that M(NDIDP) MOFs exhibit notable dispersions in the conduction band minima for efficient $n$-type charge transport and conductivity. Strikingly, conductivity of M(NDIDP) was also found to be highly configuration-dependent, as evidenced by the shift from 1D to 2D charge transport and up to 5.7-fold increase in band dispersion (7.5fold reduction in carrier effective mass) between different structural configurations within a single material. This effectively demonstrates that electrical conductivity of M(NDIDP) MOFs can be finely tuned via application of external stimuli, making them excellent candidate materials for multifunctional electronics.

\section{Results and Discussion}

\section{Modular Design of M(NDIDP)}

Many flexible MOFs have been synthesized to date, and diverse structural motifs for framework flexibility have been implemented. One of the most common structural motifs for flexibility is the "wine rack" motif, where one-dimensional metal clusters are present as pillars and bidentate linkers serve as spokes that bind the pillars together (Figure 1a). In such MOFs, coordination bonds between the metals and linkers function as hinges, and they are collectively arranged in a manner that allows for a significant expansion and contraction of the framework. Representative MOFs of this motif are MIL-47, ${ }^{35}$ MIL-53, ${ }^{36}$ and M(BDP),${ }^{32-34}$ which are 
widely recognized for their stepwise gas adsorption behavior arising from the large variability in the pore volume. Here, it is important to note that flexibility in these MOFs is conferred by their unique metal-linker coordination environment and topologies. Hence, by performing further structural modifications without altering the coordination environment, new properties could be instilled in the wine rack MOFs whilst retaining their flexibility.
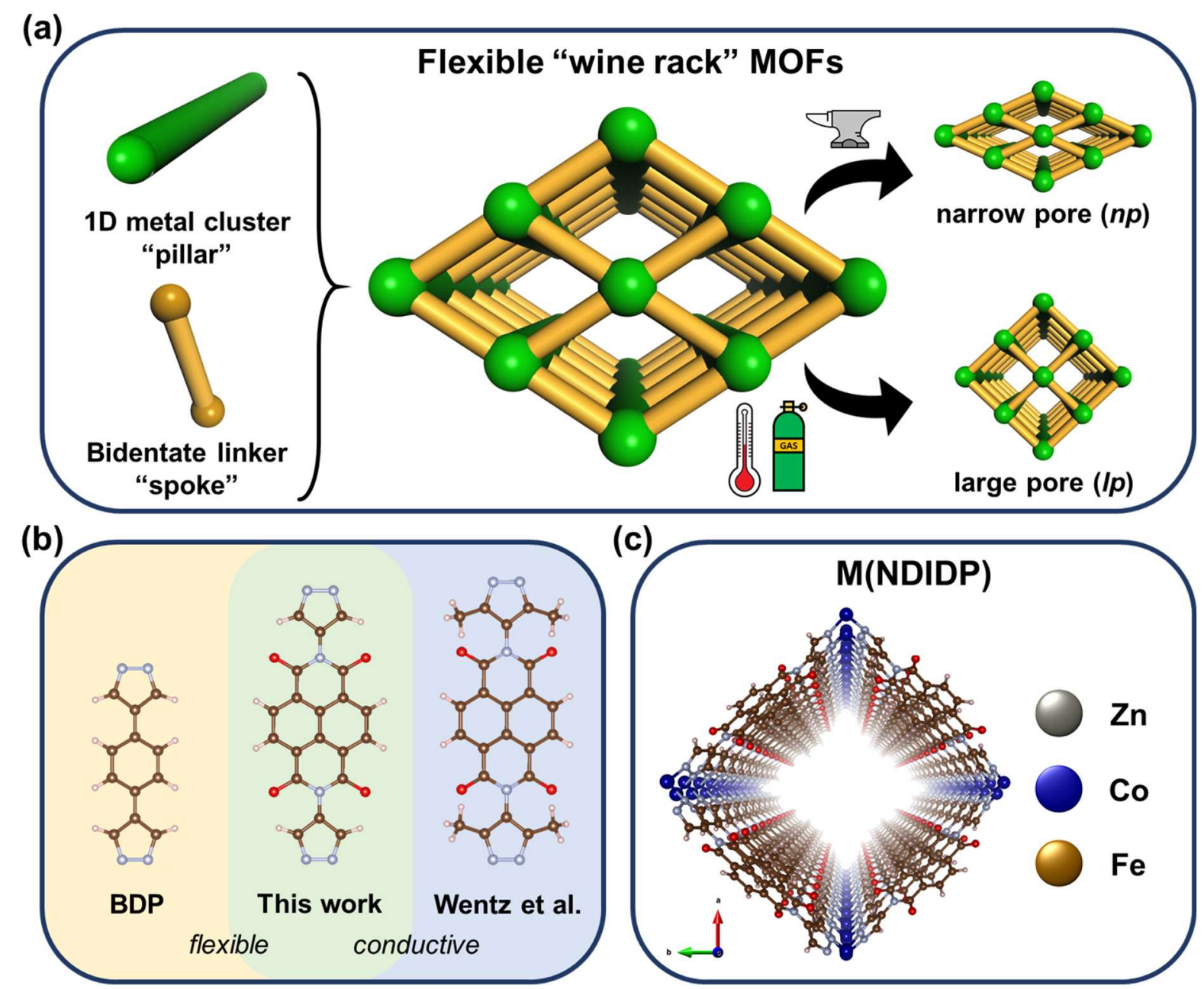

Figure 1. (a) Flexible MOFs with wine rack structural motif built from 1D metal clusters and bidentate organic linkers. (b) Bidentate pyrazolate linkers considered for MOF construction. (c) Structure of the newly proposed M(NDIDP) MOFs shown along with the metals that have been used for the construction of the three separate MOFs.

Recently, Wentz et al. reported switchable electrical conductivity in a zinc pyrazolate MOF via reversible $n$-doping of the framework. ${ }^{37}$ Interestingly, this MOF is isoreticular to M(BDP) but displays two key structural differences: redox-active NDI moieties are present as central moieties in the linkers, and the pyrazolate binding moieties are methylated at the 3 and 5 positions (Figure 1b). Electrical conductivity is effectively conferred to the MOF by the 
presence of NDI moieties, as they are stacked together in a regular fashion to facilitate CT. However, the methyl groups of pyrazolate binding moieties are found to significantly hinder the MOF from partaking in large amplitude structural transitions despite the wine rack motif (see Supporting Information). As such, we conjectured that modular substitution of benzene with NDI in M(BDP) could induce electrical conductivity in the resulting MOF while retaining the framework flexibility, thereby allowing for stimuli-controlled conductivity.

Resultantly, MOFs isoreticular to M(BDP) yet containing NDI moieties were created in silico (Figure 1c). First, a four-linker unit cell of M(BDP) was procured from the Cambridge Structural Database (refcode: QUPZAE and QUPZIM). The central benzene moieties of the BDP linkers were then computationally substituted with NDI, resulting in the proposed MOF structures. In the process, the original unit cell of $\mathrm{M}(\mathrm{BDP})$ was expanded along the linker directions to easily accommodate the redox-active NDI moieties. Three different MOFs were built from $\mathrm{Zn}, \mathrm{Co}$, and Fe metal pillars, which allows further investigation of how different metal ions affect the resulting properties. The new MOFs are herein referred to as M(NDIDP) $(\mathrm{M}=\mathrm{Zn}, \mathrm{Co}, \mathrm{Fe}, \mathrm{NDIDP}=N, N$-di(1H-pyrazol-4-yl)-1,4,5,8-naphthalenediimide $)$. It must be stated that the NDI-containing linkers incorporated into M(NDIDP) have yet to be synthesized. Nonetheless, judging from the abundance of similar NDI-containing organic linkers and their successful incorporation into MOFs,${ }^{38-42}$ we expect the linker synthesis to become possible in the near future.

\section{Flexibility of M(NDIDP): Energy vs. Volume Profile}

Next, calculations were performed to determine whether framework flexibility was successfully manifested in our M(NDIDP) MOFs. As previously discussed, flexibility targeted in this work is characterized by large amplitude expansion and contraction of the framework. In such a case, structural degrees of freedom exhibited by the MOF would allow a wide range of energetically accessible configurations, often with multiple metastable phases present as minima in the (free) energy profile. Conversely, a rigid MOF would be characterized by a narrow, parabolic energy profile with a single global minimum. Note that the expansion and contraction of flexible MOFs often incur vast differences in their unit cell volume. Hence, energy-versus-volume, or $E(V)$ profiles for each of the M(NDIDP) MOFs were obtained at the DFT level of theory (see Figure 2). Here, each point corresponds to an optimized structural 
configuration at the given unit cell volume, plotted according to its energy difference from the global energy minimum. The $E(V)$ profiles of all three M(NDIDP) MOFs exhibit a significantly wide range of energetically accessible configurations (i.e., $\Delta E<2 \mathrm{eV}$ ) bounded by the lower and higher volume limits. At these volume extrema, energy rises steeply upon further structural compression or expansion that surpasses the range of motion allowed by the flexible MOFs. Provided that a multitude of energetically accessible configurations are observed over a wide volume range as opposed to the case of rigid MOFs, ${ }^{43-45}$ one can conclude that M(NDIDP) MOFs have the potential to show framework flexibility.

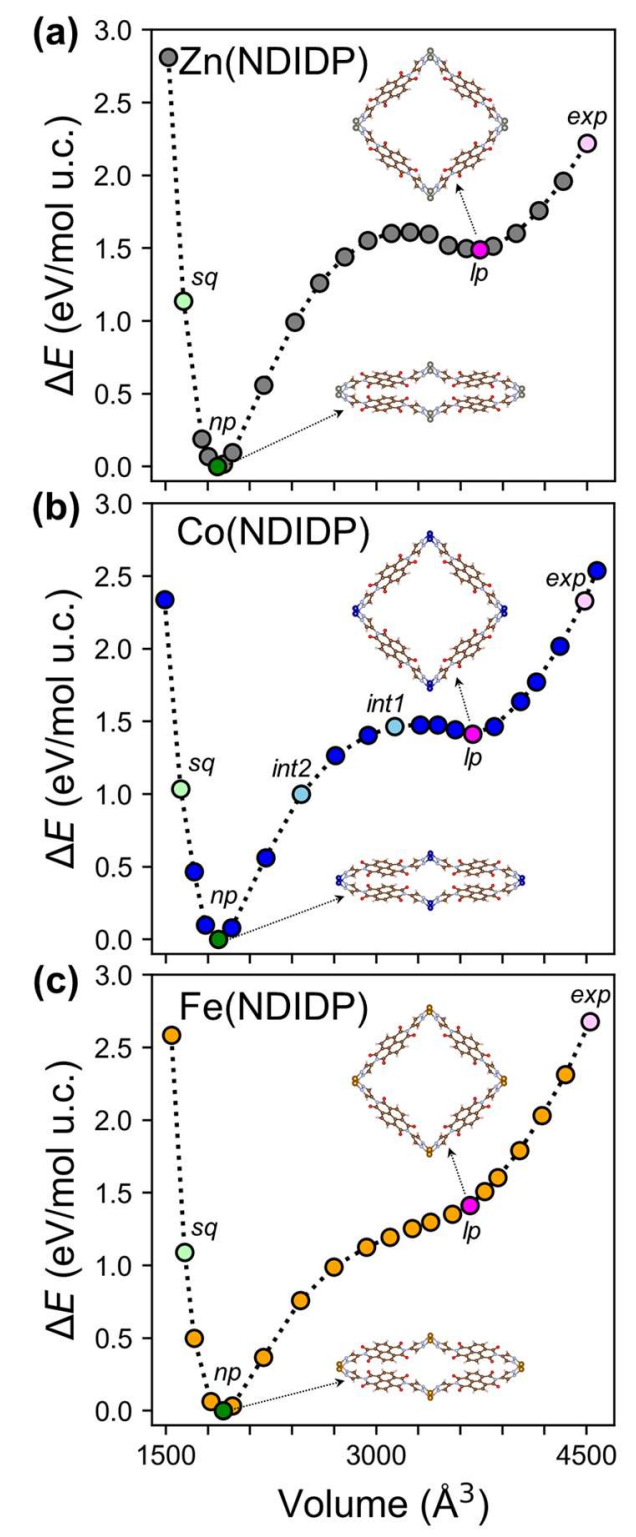

Figure 2. $E(V)$ profiles of (a) Zn(NDIDP), (b) Co(NDIDP), and (c) Fe(NDIDP). Dark green and magenta points correspond to the (meta)stable $n p$ phase and $l p$ phase of the MOFs, for which the corresponding MOF configurations are shown as insets. Lighter green and lighter 
magenta points indicate the squeezed $(s q)$ and expanded (exp) configurations discussed later. Light blue points for Co(NDIDP) indicate intermediate (int1 and int2) configurations also discussed later. Further structural information of the highlighted phases is shared in Table S1.

The global energy minima of the three M(NDIDP) MOFs are found in the lower volume regime, at $V=1867.6,1875.2$ and $1908.3 \AA^{3}$ for Zn, Co, and Fe(NDIDP) respectively. MOF configurations at these volumes (see Figure 2 insets and Figures S1-S3) correspond to the narrow pore ( $n p)$ phase, and hence all three M(NDIDP) MOFs are expected to energetically favor existing in the $n p$ phase over other structural configurations at $0 \mathrm{~K}$. Interestingly, another energy minimum is found at $V=3735.4 \AA^{3}$ for $\mathrm{Zn}$ (NDIDP), and also for Co(NDIDP) at $V=$ $3688.9 \AA^{3}$, which correspond to a metastable large pore (lp) phase. In the case of Fe(NDIDP), however, such an additional $l p$ energy minimum is not observed, and the $n p$ phase is the only thermodynamically stable structural configuration. Nonetheless, the $E(V)$ profile of Fe(NDIDP) is far from being strictly parabolic and displays a large, shoulder-like feature in the higher volume range. This indicates that the higher volume configurations of $\mathrm{Fe}(\mathrm{NDIDP})$ are still energetically accessible, yet these configurations cannot be stably sustained by the framework at $0 \mathrm{~K}$. Such differences in the $E(V)$ profiles can be explained by the softer binding tendency of $\mathrm{Fe}$, which would be more lenient towards configurational changes around the coordination environment. As such, one can conclude that the binding tendency of the metal determines the stability of the additional, metastable $l p$ phase that could be exhibited by the MOF. For the sake of consistency in the analyses that follow, the $V=3667.4 \AA^{3}$ configuration of Fe(NDIDP) was chosen to serve as the $l p$ phase, as it is the closest in unit cell volume to $\mathrm{Zn}$ (NDIDP) $-l p$ and Co(NDIDP)-lp .
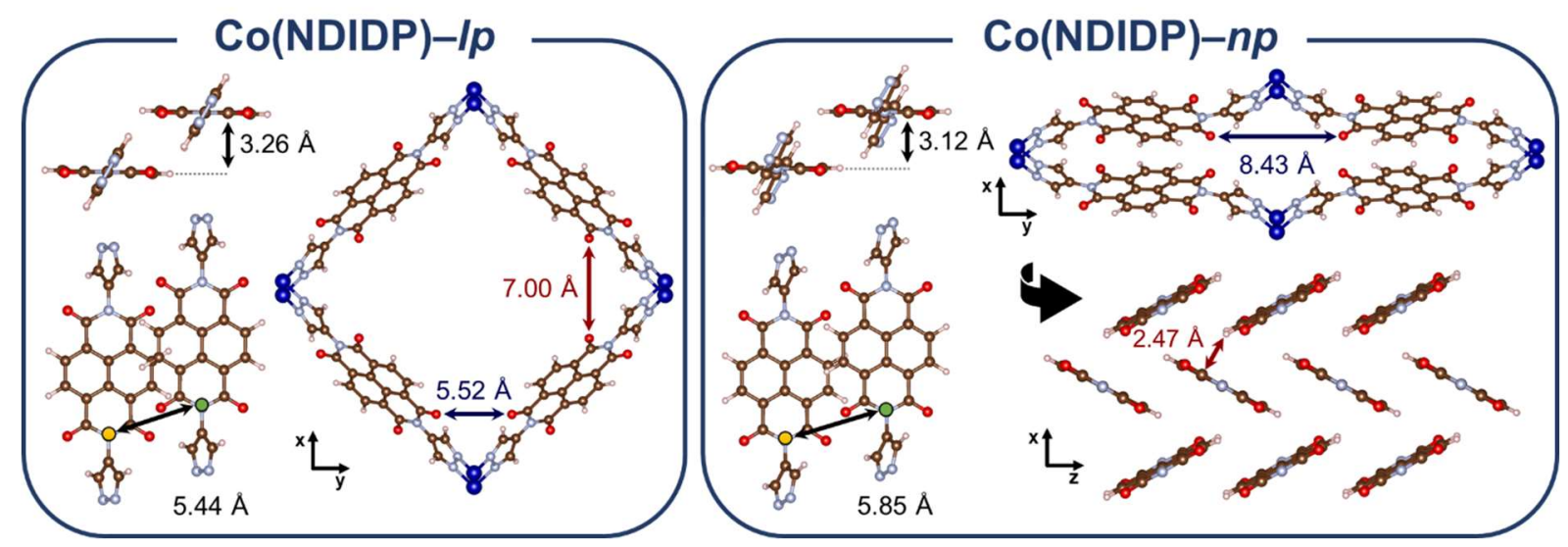

Figure 3. Internal arrangement of the NDI moieties in Co(NDIDP)-lp and Co(NDIDP) $-n p$. The parallel stacking distance (top left of each panel) and planar alignment offset (bottom left of each panel) in the $z$-direction, as well as the inter-NDI distances in the $x$ - and $y$-direction 
(right of each panel), are presented. As for the $n p$ phase, herringbone packing configuration of NDI moieties is separately shown with their edge-to-face distance.

Having installed the redox-active NDI moieties to induce CT, their relative arrangements within M(NDIDP) under the different, energetically accessible configurations were studied. Figure 3 shows the internal NDI arrangements of Co(NDIDP) in the (meta)stable $l p$ and $n p$ phases as a case study. In Co(NDIDP)-lp, NDI moieties are arranged in a paralleldisplaced manner along the $z$-axis. The stacking distance between adjacent NDI moieties is $3.26 \AA$, and the planar alignment offset is $5.44 \AA$. These distances constitute reasonable contact between NDI moieties for $\pi$ orbital overlap, which is essential for the anticipated CT. Along the $x$-axis (resp. $y$-axis), the inter-NDI distance surpasses $7.00 \AA$ (resp. $5.52 \AA$ ) at the closest contact (i.e., $\mathrm{N}-\mathrm{O}$ distance between imides of adjacent NDI), effectively prohibiting $\pi$ orbital overlap in these directions. In Co(NDIDP) $-n p$, NDI moieties retain their parallel-displaced configuration along the $z$-axis, but with notable differences from the $l p$ phase. The stacking distance between NDI moieties is decreased to $3.12 \AA$, and planar alignment is increased to $5.85 \AA$. Interestingly, contraction of the framework into the $n p$ phase also leads to the onset of edge-to-face interactions between adjacent NDIs along the $x$-axis, as evidenced by the short NDI-H distances of $2.47 \AA$. The inter-NDI distances along the $y$-axis surpass $8.43 \AA$, further prohibiting orbital overlap in this direction. Hence, Co(NDIDP)- $n p$ exhibits two-dimensional, herringbone packing of NDI in the $x z$-plane, where the orbital overlap between NDI moieties could extend along the two packing dimensions. As shown in Table S2, similar internal NDI arrangements in the $l p$ and $n p$ phases are also found in Zn(NDIDP) and Fe(NDIDP). All in all, vastly distinct internal NDI arrangements are observed in the different structural configurations of $\mathrm{M}(\mathrm{NDIDP})$, which could give rise to notably different CT properties.

\section{Flexibility of M(NDIDP): Pressure vs. Volume Equation of State}

For a MOF to be truly flexible, transitions between different metastable phases and structural configurations must be possible. Hence, the thermodynamic feasibility of structural transitions in M(NDIDP) MOFs was subsequently investigated. In doing so, we specifically focused on the pressure-induced structural transitions in M(NDIDP) materials, where an external pressure or mechanical stress is used as the stimulus. To this end, DFT-based force fields were derived using the QuickFF software for each of the three M(NDIDP) MOFs and their pressure-versus-volume or $P(V)$ equations of state at $T=300 \mathrm{~K}$ were derived following 
the established protocol, ${ }^{46,47}$ resulting in the profiles shown in Figure 4.
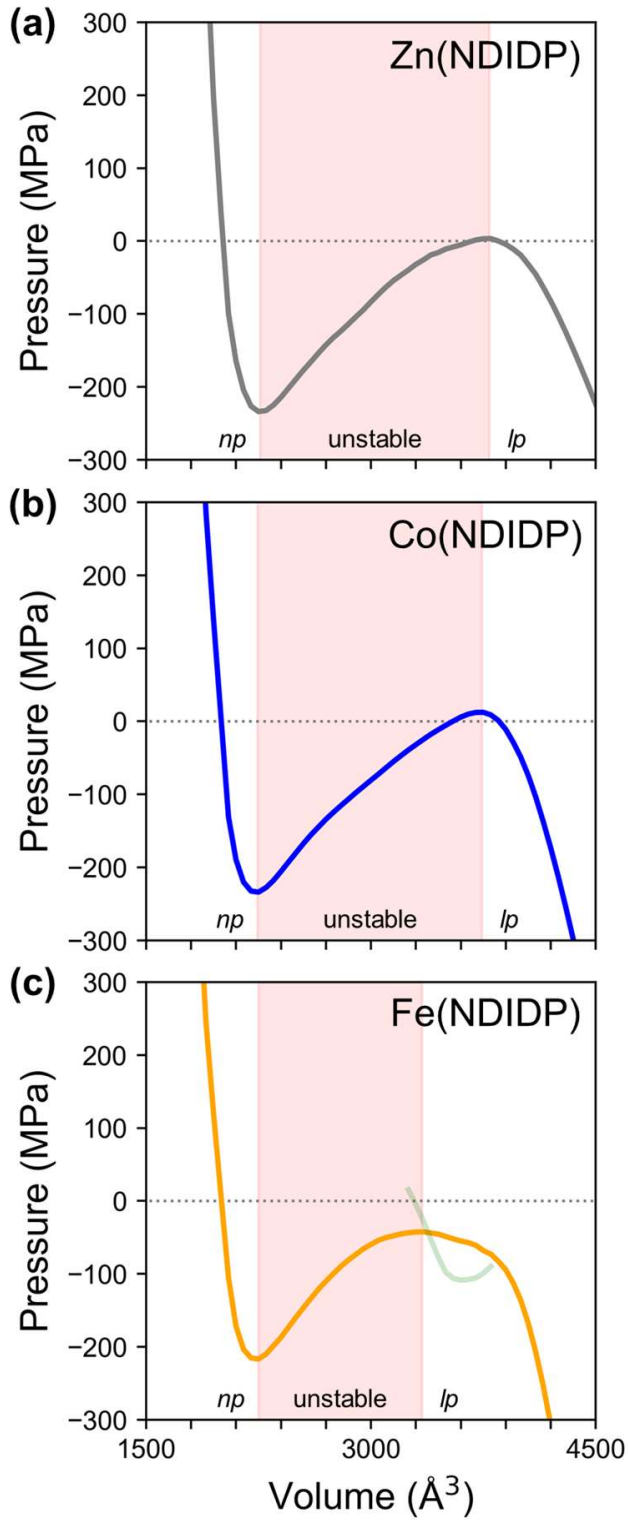

Figure 4. $P(V)$ equations of state at $T=300 \mathrm{~K}$ for (a) $\mathrm{Zn}(\mathrm{NDIDP})$, (b) Co(NDIDP), and (c) $\mathrm{Fe}(\mathrm{NDIDP})$. Gray dotted line pertaining to the ambient pressure is drawn at $0.1 \mathrm{MPa}$. Regions shaded in red indicate the mechanically unstable volume range as determined by positive $\partial P / \partial V$ values. In the case of Fe(NDIDP), a secondary partial $P(V)$ curve is shown in light green. This corresponds to the sheared configurations that have deviated away from the main mode of flexibility along the $x$-axis, as discussed in more detail in the Supporting Information.

Owing to their structural motif, the three M(NDIDP) structures exhibit $P(V)$ equations of state similar to other wine rack MOFs. ${ }^{27,46,48}$ Specifically, an intermediate, mechanically unstable region with positive $\partial P / \partial V$ values is found to separate two mechanically stable $n p$ and lp branches, which extend toward low volumes (below $2250 \AA^{3}$ ) and high volumes (above 3340 
$\AA^{3}$ for Fe(NDIDP), $3740 \AA^{3}$ for Co(NDIDP), and $3790 \AA^{3}$ for Zn(NDIDP)), respectively. Each point on these two mechanically stable branches can, in principle, be thermodynamically stabilized by applying a suitable isotropic stress, either positive or negative in sign, given by the pressure value at the specific volume point. Provided the presence of two separate, mechanically stable branches in the $P(V)$ equation of state, one can conclude that framework flexibility has been successfully manifested in M(NDIDP) MOFs at room temperature. At atmospheric pressure, i.e. $P=0.1 \mathrm{MPa}$, two (meta)stable intersections with negative $\partial P / \partial V$ values are found for $\mathrm{Zn}$ (NDIDP) and Co(NDIDP), whereas only one is found in the lower volume range for Fe(NDIDP). These results are in accordance with the DFT-derived $E(V)$ profiles, as they confirm the thermodynamic (meta)stability of both $l p$ and $n p$ phases under ambient conditions for Zn(NDIDP) and Co(NDIDP). Correspondingly, in Fe(NDIDP), the $P(V)$ equation of state shows thermodynamic stability only for the $n p$ phase at atmospheric pressure.

Next, the critical pressures needed to induce structural transitions between the $l p$ and $n p$ phases were extracted. From the $P(V)$ equation of state, they can be determined by identifying the local minimum and maximum in the pressure profile. In $\mathrm{Zn}$ (NDIDP), the equation of state shows a pressure minimum at $-234 \mathrm{MPa}$, and a maximum at $3 \mathrm{MPa}$, which can be identified with the $n p$-to- $l p$ and $l p$-to- $n p$ transition pressures, respectively. As for Co(NDIDP), these transition pressures are -224 and $13 \mathrm{MPa}$. For the softer Fe(NDIDP) MOF, these pressures amount to -217 and $-42 \mathrm{MPa}$. Hence, while the $l p$-to-n $n$ transition in either $\mathrm{Zn}$ (NDIDP) and Co(NDIDP) can be easily induced by applying an external pressure of 3 and $13 \mathrm{MPa}$, the same transition in Fe(NDIDP), as well as the $n p$-to- $l p$ transitions in all three materials, would require negative pressures. Note that such negative pressures are not yet practically achieved in MOFs. Nonetheless, one can envision that finite pulling forces of corresponding magnitude (applied via embedding the MOF into a flexible membrane, etc.) would induce the $n p$-to-l $l p$ transition.

An interesting feature found in the $P(V)$ equations of state is an anomaly in the curve around $V=\sim 1650 \AA^{3}$, at elevated pressures of ca. 1.5 GPa (Figure S4). Structural configurations to the right of the anomaly (higher volume, Figure S5) closely resemble the $n p$ phase and retain the 4-fold rotational symmetry around the 1D metal clusters. On the other hand, configurations to the left of the anomaly (lower volume, Figure S6) are all rotationally distorted around the metal clusters, and the 4-fold symmetry is reduced to 2-fold symmetry. This means that at higher pressures, the framework becomes rotationally distorted to alleviate 
the stress being exerted. Note that the reduction of rotational symmetry interestingly improves the parallel alignment between adjacent NDI moieties, which could be more favorable for $\pi$ orbital overlap and CT. As such, it can be conjectured that by applying pressures of $\sim 1.5 \mathrm{GPa}$ or higher, rotational distortions could be induced in M(NDIDP), which could potentially lead to further enhancements in their electrical conductivity. Here, we additionally note that in the case of $\mathrm{Fe}(\mathrm{NDIDP})$, a separate $P(V)$ curve was observed for the volume range between 3250 and $3800 \AA^{3}$ (see Supporting Information). Corresponding structural configurations are shown in Figure S7, and they are all found to be sheared in the $z$-axial direction compared to the main configurations. As these configurations deviate away from the main mode of flexibility under interest here (i.e., expansion/contraction based on the wine rack motif), we did not further investigate them. Nonetheless, this hints that other modes of flexibility could also exist in M(NDIDP), giving rise to more structural configurations with their own, unique NDI arrangements and resulting CT properties.

\section{Electrical Conductivity of M(NDIDP) and Configuration-Dependent CT}

Following the thorough affirmation of their framework flexibility, M(NDIDP) MOFs were analyzed to determine whether or not electrical conductivity has been successfully induced. In order for a MOF to harness conductivity, it must be engineered with long-range CT pathways for electron or hole transport. ${ }^{11,12}$ As for M(NDIDP), the installation of redox-active NDI as the central linker moiety and their closely packed arrangement within the framework are expected to provide the necessary CT pathways. The presence of valid CT pathways in a MOF can be deduced from its electronic band structure, where dispersive frontier bands would be present from significant orbital overlap between the proposed pathway components. Such band dispersions give rise to small effective carrier mass during band-like charge transport, which then guarantees high carrier mobility for observable conductivity. As such, DFT electronic band structures of each of the M(NDIDP) MOFs were calculated and analyzed to determine whether our modular design approach has successfully secured such pathways in these materials.

Electronic band structures and projected density of states (PDOS) of Co(NDIDP) are presented in Figure 5, and those of Zn(NDIDP) and Fe(NDIDP) are shown in Figures $\mathbf{S 8}$ and S9. Band structures are drawn along the $\Gamma-X$ and $\Gamma-Z$ high symmetry paths, corresponding to 
the directions in which close-packing of the NDI units was previously observed. Given their dissimilar NDI arrangements, calculations were performed for both $l p$ and $n p$ phases. Remarkably, band structures of all three M(NDIDP)s in both phases exhibit notable dispersion in their conduction band minimum (CBM), which is ideal for n-type CT. From the PDOS and band-decomposed charge density plots (Figure S10), sole contributors to the CBM are revealed to be the $\mathrm{C}$ and $\mathrm{O}$ atoms of the NDI moieties. This shows that the CBM of M(NDIDP) MOFs is exclusively composed of the orbitals provided by NDI. Dispersion of the CBM hence arises from the orbital overlap between closely packed NDI units, which effectively completes the CT pathways in M(NDIDP) MOFs as intended by our modular design approach. Band gaps $\left(E_{g}\right)$ of the MOFs under the two phases are found well within the semiconducting range, with values ranging between 0.16 and $2.39 \mathrm{eV}$ (see Figures 5, S8, and S9). As such, all three MOFs in both $l p$ and $n p$ phases are predicted to show electrical conductivity, which can be triggered by populating the CBM with electrons via excitation or $n$-doping of the system. Note that large dispersions are also present in the valence band maximum (VBM) in some cases, especially for Fe(NDIDP). Such VBM dispersions are suspected to be artefacts of the DFT methods adopted in our study, and should not be directly interpreted as indications of efficient hole transport (see Supporting Information).
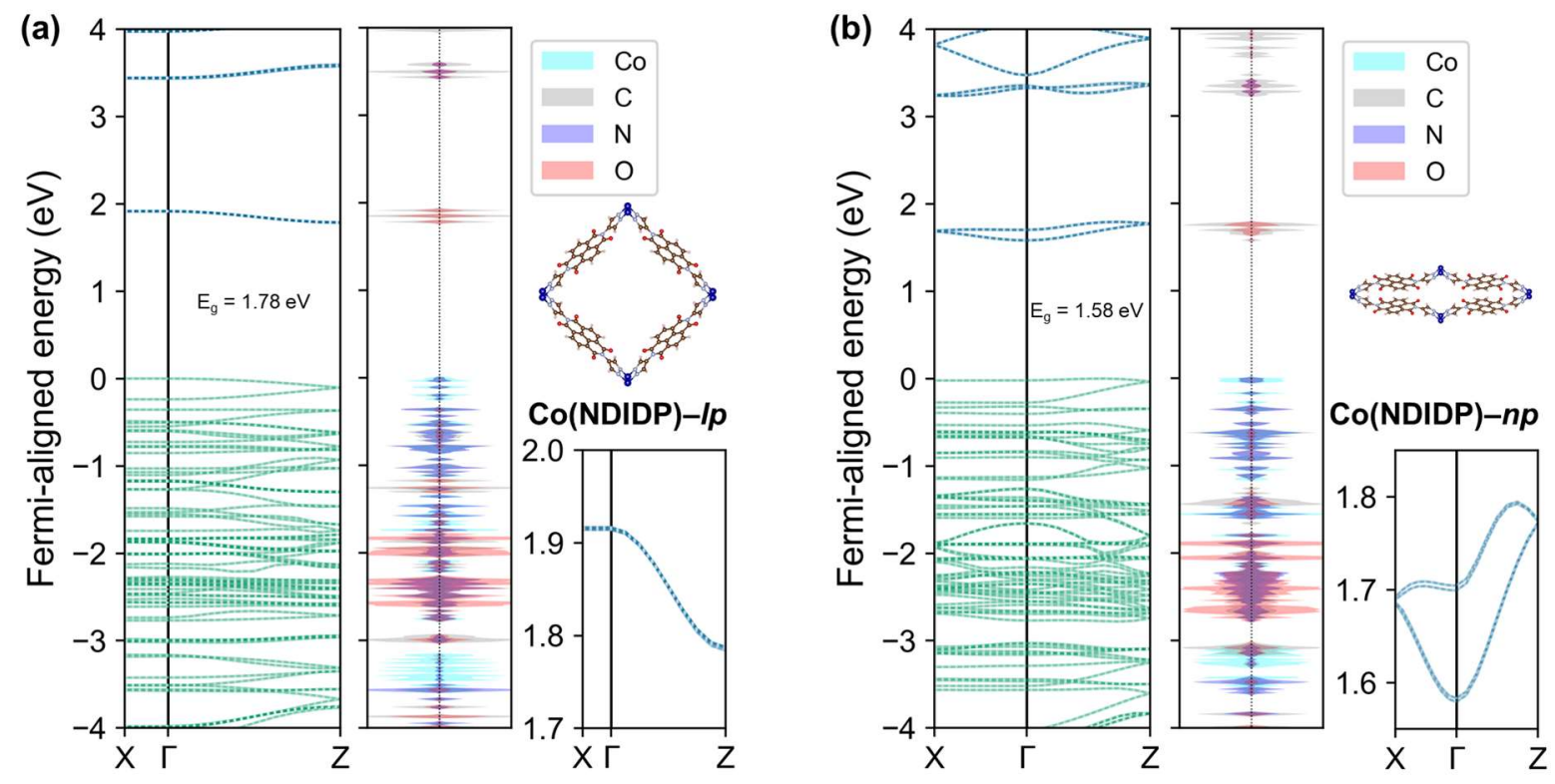

Figure 5. Electronic band structures and projected density of states (PDOS) of (a) Co(NDIDP)$l p$ and (b) Co(NDIDP)-np. Conduction band minima corresponding to NDI are shown separately in the bottom right. Green bands are filled valence bands, and blue bands are empty conduction bands. Solid and dotted bands (with lighter color) correspond to up and down spins. 
In M(NDIDP)- $l p$, dispersion of the CBM is limited to the $\Gamma-\mathrm{Z}$ path, and the band remains strictly flat along the $\Gamma-\mathrm{X}$ path. This is in accordance with the internal NDI arrangement observed in the $l p$ phase, where parallel-displaced packing along the $z$-axis was found. As such, CT in M(NDIDP)-lp is expected to propagate one-dimensionally along this axis. Dispersion is measured to be 83, 130, and $212 \mathrm{meV}$ for $\mathrm{Zn}, \mathrm{Co}$, and Fe(NDIDP)-lp, respectively. Here, we note the increasing trend of CBM dispersion in the presented order of MOFs. This is also attributed to the different binding tendencies of metal ions, which becomes softer from $\mathrm{Zn}$ to $\mathrm{Co}$, then Fe. Softer metal ions would allow the MOF to further distort its structure, better optimizing the orbital overlap between stacked NDI moieties. This is indeed corroborated by their internal NDI arrangements (see Table S2), where the adjacent NDI moieties become more parallelly aligned in the same order. Electron effective mass at the CBM edges $\left(m_{\mathrm{e}}{ }^{*}\right)$ were also calculated and are presented in Table 1. Although $m_{\mathrm{e}}{ }^{*}$ values along the $\Gamma-\mathrm{Z}$ path in M(NDIDP)-lp are relatively high, they still constitute significant improvements over the non-conducting case (i.e. $m_{\mathrm{e}}{ }^{*} \rightarrow \infty$ ), and hence effectively validating the presence of CT pathways.

Table 1. CBM dispersion values and corresponding effective masses of electron $\left(m_{\mathrm{e}}{ }^{*}\right)$ obtained from the band structures of $\mathrm{M}(\mathrm{NDIDP})-l p$ and $\mathrm{M}(\mathrm{NDIDP})-n p$. ( $m_{0}$ : electron rest mass)

\begin{tabular}{|c|c|c|c|c|}
\hline \multirow{2}{*}{ M(NDIDP) } & \multicolumn{2}{|c|}{ large pore $(l p)$} & \multicolumn{2}{c|}{ narrow pore $(n p)$} \\
\cline { 2 - 5 } & $\Gamma-\mathrm{X}$ & $\Gamma-\mathrm{Z}$ & $\Gamma-\mathrm{X}$ & $\Gamma-\mathrm{Z}$ \\
\hline \multirow{2}{*}{$\mathrm{Zn}$} & & $83 \mathrm{meV}$ & $73 \mathrm{meV}$ & $200 \mathrm{meV}$ \\
& & $m_{\mathrm{e}}{ }^{*}=4.37 m_{0}$ & $m_{\mathrm{e}}{ }^{*}=3.02 m_{0}$ & $m_{\mathrm{e}}{ }^{*}=1.51 m_{0}$ \\
\cline { 3 - 5 } $\mathrm{Co}$ & $\sim 0 \mathrm{meV}$ & $130 \mathrm{meV}$ & $121 \mathrm{meV}$ & $211 \mathrm{meV}$ \\
& $m_{\mathrm{e}}^{*} \rightarrow \infty$ & $m_{\mathrm{e}}{ }^{*}=2.76 m_{0}$ & $m_{\mathrm{e}}{ }^{*}=2.36 m_{0}$ & $m_{\mathrm{e}}{ }^{*}=1.64 m_{0}$ \\
\cline { 3 - 5 } $\mathrm{Fe}$ & & $212 \mathrm{meV}$ & $403 \mathrm{meV}$ & $633 \mathrm{meV}$ \\
& & $m_{\mathrm{e}}{ }^{*}=1.71 m_{0}$ & $m_{\mathrm{e}}{ }^{*}=1.17 m_{0}$ & $m_{\mathrm{e}}{ }^{*}=0.73 m_{0}$ \\
\hline
\end{tabular}

In discussing the case of M(NDIDP)- $n p$, we first note the apparent splitting of the $\mathrm{CBM}$ at the $\Gamma$ point. Such phenomenon arises from the presence of two NDI moieties along the $x$-axis within the chosen M(NDIDP) unit cell, which come in contact with one another in the $n p$ phase (see Figure 3). Consequently, two pairs of NDI moieties are formed, and their orbital interactions within the cell cause the CBM to undergo band splitting at the $\Gamma$ point. Note that a single NDI moiety serves as one electronic unit, yet the unit cell of $\mathrm{M}(\mathrm{NDIDP})-n p$ contains 
two electronic units along both $x$ and $z$-axis (see Supporting Information). Therefore, the CBM should rather be understood as having been folded due to the presence of multiple electronic units within the unit cell. ${ }^{49}$ This interpretation is further substantiated by the convergence of $\mathrm{CBM}$ and $\mathrm{CBM}+1$ band energies observed at the $\mathrm{X}$ and $\mathrm{Z}$ symmetry points. As such, set of bands all corresponding to the lowest unoccupied orbitals of NDI were collectively regarded as the CBM, and band dispersion and $m_{\mathrm{e}}{ }^{*}$ were calculated accordingly.

The CBM of M(NDIDP) $-n p$ along the $\Gamma-\mathrm{Z}$ path shows significant dispersions of 200 , 211, and $633 \mathrm{meV}$ for $\mathrm{Zn}, \mathrm{Co}$, and Fe(NDIDP)-np, respectively, which constitutes an up to 3fold increase compared to the $l p$ phase. Such enhancements are attributed to the tighter packing of NDI along the $z$-axis (see Table S2), which drastically improves the orbital overlap between adjacent NDI moieties. Interestingly, band dispersion is newly observed along the $\Gamma-X$ path as well in all three MOFs. This confirms that 2D herringbone packing of NDI exhibited by $\mathrm{M}(\mathrm{NDIDP})-n p$ can provide sufficient orbital overlap along both dimensions, resulting in twodimensional CT. Dispersion along the $\Gamma-\mathrm{X}$ path are 73, 121 and $403 \mathrm{meV}$ for $\mathrm{Zn}, \mathrm{Co}$, and $\mathrm{Fe}(\mathrm{NDIDP})-n p$, which are comparable to the $\Gamma-\mathrm{Z}$ path in $\mathrm{M}(\mathrm{NDIDP})-l p$. Along both high symmetry paths, the previously observed correlation between binding tendencies of metal ion and the resulting band dispersion are found to persist. Trends in the band dispersion are correspondingly carried over to $m_{\mathrm{e}}{ }^{*}$ (see Table 1), where the $m_{\mathrm{e}}{ }^{*}$ is notably reduced along the $\Gamma-\mathrm{Z}$ path compared to M(NDIDP)- $l p$, and finite $m_{\mathrm{e}}{ }^{*}$ values are newly observed along the $\Gamma-\mathrm{X}$ path. The smallest $m_{\mathrm{e}}{ }^{*}$ of $0.73 m_{0}$ obtained for Fe(NDIDP) $-n p$ along the $\Gamma-\mathrm{Z}$ path is noteworthy, as it is smaller than the electron rest mass and comparable to the values reported for other known conductive MOFs. ${ }^{13,50,51}$ Note that $\mathrm{E}_{\mathrm{g}}$ of Co(NDIDP) decreases from 1.78 to $1.58 \mathrm{eV}$ as the MOF transitions from $l p$ to $n p$ phase. Similar reduction of $E_{\mathrm{g}}$ is observed for the other two MOFs (see Figures S8 and S9), where $E_{g}$ decreases from 2.39 to $2.20 \mathrm{eV}$, and from 0.54 to $0.16 \mathrm{eV}$ in $\mathrm{Zn}$ and $\mathrm{Fe}(\mathrm{NDIDP})$, respectively. These changes in $\mathrm{E}_{\mathrm{g}}$ are consistent with what was previously reported for MIL-47 and MIL-53. ${ }^{31}$

Results thus far demonstrate that while significant conductivity is predicted for all three M(NDIDP)s, their precise CT properties vary drastically depending on their structural configurations. Between the $l p$ and $n p$ phases, differences in the CT properties are characterized by an up to 3-fold increase in band dispersion (2.9-fold reduction in $\left.m_{\mathrm{e}}{ }^{*}\right)$ and a transition from $1 \mathrm{D}$ to $2 \mathrm{D}$ transport. Such differences are explained by changes in the internal NDI arrangement, 
which progresses from $1 \mathrm{D}$ parallel-displaced packing to $2 \mathrm{D}$ herringbone packing as the MOF transitions from the $l p$ to the $n p$ phase.

\section{Further Exploration of Configuration-Dependent CT}

Based on the findings thus far, we conclude that two material properties, namely flexibility and conductivity, have been successfully instilled and synergistically combined in M(NDIDP), resulting in tunable electrical conductivity. Next, one could envisage that conductivity of M(NDIDP) could be further tuned by taking advantage of their flexibility and exploring more of their allowed structural configurations. Hence, additional structural configurations of $\mathrm{M}(\mathrm{NDIDP})$ were selected and analyzed for their unique CT properties. Two intermediate configurations located between the $l p$ and $n p$ phases, herein referred to as int 1 and int2, were first extracted (light blue points in Figure 2). Additionally, "squeezed" ( $s q$ ) and "expanded" (exp) configurations were obtained as structures smaller than the $n p$ phase or larger than the $l p$ phase, respectively, whilst exhibiting an energy difference of less than $1.5 \mathrm{eV}$ from the corresponding phases at $0 \mathrm{~K}$ (light green and light magenta points in Figure 2). While these configurations are not thermodynamically accessible under ambient conditions, they are still of considerable interest since external stimuli (e.g., guest loading, mechanical stress, isotropic pulling) could effectively lead to their stabilization. Band structures were subsequently calculated for the chosen configurations in Co(NDIDP) (Figure S11 and S12), and the results are presented in Figure 6 and S13.

In Co(NDIDP)- $\exp$ (Figure 6a), where the MOF is further expanded past the $l p$ phase (Figure 6b), significant enhancement in $\mathrm{CT}$ is interestingly observed. While the CBM remains flat along the $\Gamma-\mathrm{X}$ path, band dispersion along the $\Gamma-\mathrm{Z}$ path increases from 130 to $280 \mathrm{meV}$, and $m_{\mathrm{e}}{ }^{*}$ decreases from $2.76 m_{0}$ to $0.91 m_{0}$. Eg is found to be $1.73 \mathrm{eV}$ (see Figure S13), which is slightly lower than Co(NDIDP)-lp. It is also noted that energy of the CBM at the $\mathrm{Z}$ point is higher than the energy at the $\Gamma$ point, whereas the inverse trend was observed in the $l p$ phase. These phenomena are collectively attributed to the reconfiguration of NDI moieties accompanying further framework expansion (see Table S2). The internal NDI arrangement of Co(NDIDP)-exp exhibits a notable decrease in the $z$-axial stacking distance from $3.26(l p)$ to $3.12 \AA$ (exp), which improves the orbital overlap between NDI moieties and therefore explains the increased band dispersion. Concurrently, the planar alignment offset surges from 5.44 ( $l p$ ) 
to $6.63 \AA$ (exp). Such a large difference in NDI alignment is significant enough to induce a change of sign in the transfer integral between adjacent moieties, hence inverting the energy trend along the $\Gamma-\mathrm{Z}$ path. As presented in Figure S14, notably enhanced CT properties are consistently observed in $\mathrm{Zn}(\mathrm{NDIDP})-\exp$ and Fe(NDIDP)-exp as well (structures shown in Figure S15) compared to their $l p$ counterparts. This effectively demonstrates that if M(NDIDP) can be further expanded past the $l p$ phase, for instance by an isotropic pulling of the material, their electrical conductivity would be notably enhanced.
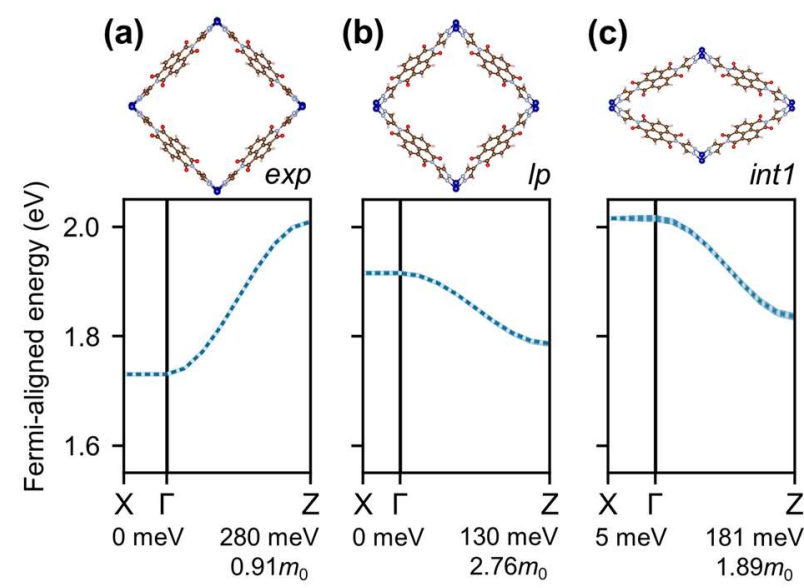

(d)

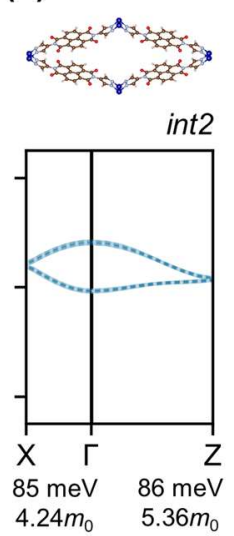

(e)

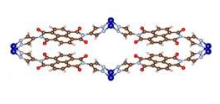

(f)

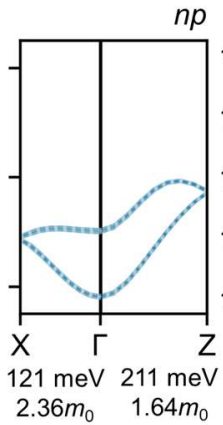

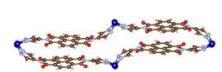

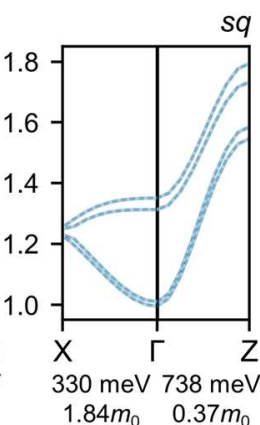

Figure 6. Evolution of the conduction band minimum (CBM) of Co(NDIDP) upon decreasing volume under all of the structural configurations considered. Full electronic band structures of the additional structural configurations (exp, int 1, int $2, s q$ ) are shared in Figure S13. Solid (blue) and dotted bands (lighter blue) correspond to up and down spins.

Next, two intermediate int 1 and int 2 configurations (Figures $\mathbf{6 c}$ and $\mathbf{6 d}$ ) between the metastable $l p$ and $n p$ phases were analyzed, which allow one to study the evolution of the CBM as $\mathrm{Co}(\mathrm{NDIDP})$ undergoes the $l p$-to- $n p$ transition. Along the $\Gamma-\mathrm{X}$ path, dispersion is found to monotonically increase as follows: $0 \mathrm{meV}(l p), 5 \mathrm{meV}$ (int 1), $85 \mathrm{meV}$ (int2), and $121 \mathrm{meV}(n p)$. This trend is straightforwardly explained by the effect of framework contraction on the internal NDI arrangement of Co(NDIDP) (see Tables S2). Pairs of NDI moieties along the $x$-axis become closer to each other from one configuration to the next, leading to the gradual onset of their orbital overlap and $\mathrm{CT}$ in the $x$-axial direction. Along the $\Gamma-\mathrm{Z}$ path, band dispersions are $130 \mathrm{meV}($ lp), $181 \mathrm{meV}$ (int1), $86 \mathrm{meV}$ (int2), and $211 \mathrm{meV}(n p)$. Curiously, band dispersion decreases in Co(NDIDP)-int2 while the other three configurations show an increasing trend. This can be attributed to the large $z$-axial stacking distance of $3.6 \AA$ observed in Co(NDIDP)int2 as opposed to $3.26 \AA(l p), 3.40 \AA$ (int 1$)$, and $3.12 \AA(n p)$, which has significantly increased to better accommodate the pair interactions newly taking place in the $x$-axial direction. These 
results demonstrate that correlation between the structural transition of M(NDIDP) and the resulting $\mathrm{CT}$ properties is nonlinear and rather complex. Nonetheless, a gradual transition from 1D to $2 \mathrm{D}$ transport is undoubtedly observed, establishing that the conductivity of M(NDIDP) MOFs can be finely tuned by accessing the intermediate configurations between the $l p$ and $n p$ phases.

Remarkably, contraction of Co(NDIDP) past the $n p$ phase (Figure 6e) to Co(NDIDP)$s q$ (Figure 6f) also results in vastly improved CT properties. In Co(NDIDP)-sq, CBM dispersion is measured to be $330 \mathrm{meV}$ and $738 \mathrm{meV}$ along the $\Gamma-\mathrm{X}$ and $\Gamma-\mathrm{Z}$ paths. These values constitute substantial CT enhancement over the case of Co(NDIDP)- $n p$, especially along the $\Gamma-\mathrm{Z}$ path where the dispersion has increased by a factor of 3.5. Large dispersion in the CBM leads to significantly smaller $m_{\mathrm{e}}{ }^{*}$, where the effective mass is reduced from $2.36 m_{0}$ to $1.84 m_{0}$ along the $\Gamma-\mathrm{X}$ path, and from $1.64 m_{0}$ to $0.37 m_{0}$ along the $\Gamma-\mathrm{Z}$ path. $0.37 m_{0}$ is one of the lowest carrier effective masses that has been observed in a MOF, to the best of our knowledge. $\mathrm{E}_{\mathrm{g}}$ is significantly decreased from $1.58 \mathrm{eV}(n p)$ to $1.00 \mathrm{eV}(s q)$ (see Figure S13). Again, such markedly improved CT properties are associated with the internal NDI arrangement of Co(NDIDP)- $s q$ (see Table S2). In this structural configuration, NDI moieties are even more tightly packed together: the $z$-axial stacking distance is reduced from 3.12 to $2.14 \AA$, and the NDI-H distance is also reduced from 2.47 to $2.16 \AA$. This effectively maximizes the orbital overlap between adjacent NDI moieties, which further boosts CT and the resulting conductivity. Note that the extreme contraction of Co(NDIDP) into the $s q$ configuration leads to an uneven spacing between NDI moieties in the $z$-axial direction (see Figure S16), which creates an opening between the folded bands of CBM at the $\mathrm{Z}$ point. Based on these results, we conclude that the highest conductivity of Co(NDIDP) can be attained by further contracting or compressing the MOF past the $n p$ phase.

Similar CT enhancements were not observed in the readily procured $s q$ configurations of Zn(NDIDP) and Fe(NDIDP) (Figures S17 and S18). In the case of Zn(NDIDP)-sq, only a 1.33-fold increase in the band dispersion is observed compared to the $n p$ phase. As for Fe(NDIDP)-sq, band dispersion has rather diminished compared to Fe(NDIDP)- $n p$ along both symmetry paths. We attribute these differences in CT properties to the visibly different structural configuration of $\mathrm{Co}(\mathrm{NDIDP})-s q$ (see Figure $\mathbf{S 1 2}$ ) as opposed to $\mathrm{Zn}$ or Fe(NDIDP)$s q$ (see Figure S17), where a counter-clockwise rotational distortion around the 1D metal cluster is observed. Such rotational distortion around the 1D metal cluster has also been 
computationally predicted for the low volume configurations of other wine rack MOFs in previous studies. ${ }^{52-55}$ As conjectured previously, this distortion induces a much smaller angle between the edge-to-face NDI pairs compared to $\mathrm{Co}(\mathrm{NDIDP})-n p, \mathrm{Zn}(\mathrm{NDIDP})-s q$ or Fe(NDIDP)- $s q$ (see Table S2), which is highly beneficial for $\pi$-orbital overlap. As a result, notable CT enhancement of $\mathrm{Co}(\mathrm{NDIDP})-s q$ is attributed to the observed rotational distortion. Note that similarly distorted structural configurations of Zn(NDIDP) and Fe(NDIDP) were nonetheless observed from the MD simulations (see Figure S6) for lower unit cell volumes (< $1650 \AA^{3}$ ) at room temperature. Hence, it is predicted that framework contraction past the $n p$ phase could lead to the highest conductivity in all three M(NDIDP) MOFs, as long as the rotational distortion around the metal cluster simultaneously takes place. Altogether, our analysis effectively demonstrates that electrical conductivity of M(NDIDP) is highly tunable, and many other structural configurations with unique CT properties exist for these flexible MOFs.

\section{Conclusion}

In this study, we have demonstrated that framework flexibility and electrical conductivity can be synergistically combined into a single MOF via a modular design approach. The designed MOFs, M(NDIDP) with $\mathrm{M}=\mathrm{Zn}, \mathrm{Co}, \mathrm{Fe}$, show significant framework flexibility and are capable of pressure-induced structural transitions at low pressures and room temperature. M(NDIDP) MOFs also exhibit configuration-dependent CT properties, which are characterized by transition from 1D to 2D transport and up to 5.7-fold increase in band dispersion (7.5-fold reduction in effective carrier mass) between different structural configurations. As a result, tunable electrical conductivity is predicted for the M(NDIDP) MOFs, where the degree of CT in the MOF can be controlled via application of external stimuli. The design of flexible and electrically conductive MOFs performed in this work demonstrates the practicability of simultaneously inducing multiple material properties in the same MOF via the modular design approach. We anticipate that our design approach can be extended to discover more flexible and conductive MOFs for adsorptive sensing, conformal electronics, molecular switches, and many other relevant applications that may benefit from stimuliinduced changes in electrical conductivity. 


\section{Computational Methods}

\section{DFT Calculations}

Kohn-Sham density functional theory (DFT) calculations were performed to obtain the optimized configurations of each of the M(NDIDP) MOFs and analyze the resulting electronic structures. All DFT calculations in this work were carried out with the Vienna $A b$ initio Simulation Package (VASP). ${ }^{56-58}$ The projector augmented wave (PAW) method ${ }^{59,60}$ was used to describe the interactions between the ions and electrons. The exchange-correlation term was described within the generalized gradient approximation (GGA) using the Perdew-BurkeErnzerhof (PBE) functional. ${ }^{61}$ In the newly designed MOFs, it is important to accurately account for the van der Waals interactions between the NDI moieties given their high degree of aromaticity. Hence, DFT-D3 dispersion corrections with Becke-Johnson damping were employed. $^{62} \Gamma$-centered k-point grids with spacings less than $0.3 \AA^{-1}$ were used in the calculations. The kinetic energy cutoff was set at $600 \mathrm{eV}$, and self-consistent field (SCF) convergence criteria was fixed at $1 \times 10^{-6} \mathrm{eV}$. Gaussian smearing was performed with a sigma value of $0.01 \AA$. Given the presence of unpaired d-orbital electrons, spin polarization was considered for Co(NDIDP) and Fe(NDIDP). High-spin ferromagnetic ordering along each 1D metal cluster was observed to be the most stable, and hence used to report the system energies.

Geometry optimizations were performed to construct the $E(V)$ profiles of each material. In each optimization, a volume constraint was applied. That is, the total volume of the unit cell was fixed, but the internal atomic coordinates and the lattice parameters were relaxed until the energy minimum was found. Relaxation was continued until the Hellmann-Feynman forces became lower than $0.01 \mathrm{eV} / \AA$ for all the atoms. After successfully optimizing the MOF at a given volume, length of the lattice parameter $a$ was incrementally increased or decreased to provide the starting configuration for the adjacent volume point. This procedure was repeated until the entire volume range of interest was covered. Electronic band structures were subsequently calculated for the configurations of interest. While the PBE functional can provide an accurate description of the structure of these materials, it is known to underestimate the band gaps of semiconducting materials. Hence, the HSE06 hybrid functional ${ }^{63}$ was used to more accurately describe the band structures. In doing so, revised D3 damping parameters for HSE06 reported by Moellmann and Grimme were used. ${ }^{64}$ Ten equidistant k-points within the first Brillouin zone were sampled along the high symmetry paths of interest, i.e. the $\Gamma-X$ and 
$\Gamma-\mathrm{Z}$ paths. Carrier effective masses were calculated with the sumo package, ${ }^{65}$ using a leastsquares fit with five sample points and assuming non-parabolicity of the bands.

\section{Molecular Dynamics Simulations}

Force field-based molecular dynamics (MD) simulations were performed to study the pressure-induced structural transitions of M(NDIDP). To this end, for each of the DFToptimized structures, first the Hessian matrix was calculated with VASP using $0.015 \AA$ displacements and an SCF convergence criterium of $1 \times 10^{-8} \mathrm{eV}$ for improved accuracy. The $a b$ initio periodic Hessian and optimized structure were then taken as inputs to the QuickFF software ${ }^{66,67}$ to derive the covalent force field parameters for interactions including cross terms and using the force field atom types provided in Figure S19. Gaussian-like atomic charges were used to describe the electrostatic interactions, and were determined using the Minimal Basis Iterative Stockholder (MBIS) partitioning scheme as implemented in HORTON. ${ }^{68}$ To this end, GPAW calculations ${ }^{69,70}$ were performed on the optimized geometries to obtain the allelectron density necessary for the MBIS scheme. The radii of the Gaussian charges were obtained by following the fitting approach suggested by Chen and Martínez. ${ }^{71}$ Finally, the MM3 Buckingham potential ${ }^{72}$ was used to account for the van der Waals interactions.

Using these force fields, MD simulations were performed in Yaff for a variety of volumes to obtain the $P(V)$ equations of state. ${ }^{73}$ Simulations were conducted for $1 \times 1 \times 2$ supercells of the M(NDIDP) MOFs in the $\left(N, V, \boldsymbol{\sigma}_{\mathrm{a}}=\mathbf{0}, T\right)$ ensemble, where $\boldsymbol{\sigma}_{\mathrm{a}}$ refers to the

deviatoric stress component of the stress tensor. ${ }^{46}$ In this ensemble, the unit cell $V$ is constrained, but the simulation cell shape can fluctuate freely. From these simulations, the average pressure the material exerts on its environment is extracted. This average pressure equals the external pressure exerted on the material in mechanical equilibrium and can thus be used to construct the macroscopic pressure-versus-volume equations of state. A Nosé-Hoover chain thermostat $^{74,75}$ with a chain length of 3 and a 0.1 ps relaxation time was used to control the system temperature. $\sigma_{\mathrm{a}}$ was controlled using the Martyna-Tobias-Tuckerman-Klein barostat ${ }^{76}$ with a 1 ps relaxation time. The velocity Verlet integration algorithm was used with a timestep of 0.5 fs to solve the equations of motion. A distance cutoff of $15 \AA$ was used for the van der Waals interactions with a smooth cutoff. For the electrostatic interactions, an Ewald summation was performed with a real-space cutoff of $15 \AA$, a splitting parameter of $0.213 \AA^{-1}$, and 
reciprocal space cutoff of $0.32 \AA^{-1}$. Each simulation consisted of a 0.5 ns equilibration run followed by a 0.5 ns production run. The obtained $P(V)$ equations of state were locally smoothened using the Savitzky-Golay filter. ${ }^{77}$

\section{Supporting Information}

The Supporting Information is available free of charge.

Structures of M(NDIDP) in $l p$ and $n p$ phases; $P(V)$ equation of state in the low $V$ range and corresponding configurations; Secondary phase structures of Fe(NDIDP); Band structures of $\mathrm{Zn}$ (NDIDP) and Fe(NDIDP) in $l p$ and $n p$ phases; Band-decomposed charge density plots of the CBM of M(NDIDP); Additional structural configurations of Co(NDIDP) and their band structures; Structures and band structures of the exp configuration of Zn and Fe(NDIDP); NDI arrangement in the z-axial direction for Co(NDIDP)-sq; Structures and band structures of the $s q$ configuration of $\mathrm{Zn}$ and $\mathrm{Fe}$ (NDIDP); Atom type assignment in M(NDIDP) for force field generation; Lattice parameters of M(NDIDP); Internal NDI arrangements of M(NDIDP); Flexibility and conductivity of $\mathrm{Zn}(\mathrm{NDIDP})-\mathrm{CH}_{3}$; Secondary phase configurations of $\mathrm{Fe}(\mathrm{NDIDP})$; VBM dispersion in M(NDIDP); CBM splitting/folding in smaller volume configurations; Data availability (PDF)

\section{Notes}

The authors declare no competing financial interest.

\section{Acknowledgments}

S.C. thanks the National Research Foundation (NRF) and Ministry of Education (MOE) of Korea for the Global Ph.D. Fellowship (no. 2019H1A2A1075192). S.M.J.R. acknowledges support from the Fund for Scientific Research - Flanders (FWO) through a junior postdoctoral fellowship (grant no. 12T3519N). S.C. and J.K acknowledge support from the NRF of Korea grant funded by the Korean government (MSIT) (no. 2021R1A2C2003583). This research 
utilized the computing resources of KISTI National Supercomputing Center (KSC-2020-CRE0052) as well as the Stevin Supercomputer Infrastructure, the latter provided by the VSC (Flemish Supercomputer Centre), funded by Ghent University, the Research Foundation Flanders (FWO), and the Flemish Government - department EWI. The authors acknowledge Sander Borgmans for insightful discussions.

\section{References}

(1) Guillerm, V.; Kim, D.; Eubank, J. F.; Luebke, R.; Liu, X.; Adil, K.; Lah, M. S.; Eddaoudi, M. A Supermolecular Building Approach for the Design and Construction of Metal-Organic Frameworks. Chem. Soc. Rev. 2014, 43 (16), 6141-6172.

(2) Yao, Z.; Sánchez-Lengeling, B.; Bobbitt, N. S.; Bucior, B. J.; Kumar, S. G. H.; Collins, S. P.; Burns, T.; Woo, T. K.; Farha, O. K.; Snurr, R. Q.; Aspuru-Guzik, A. Inverse Design of Nanoporous Crystalline Reticular Materials with Deep Generative Models. Nat. Mach. Intell. 2021, 3 (1), 76-86.

(3) Lee, S.; Kim, B.; Cho, H.; Lee, H.; Lee, S. Y.; Cho, E. S.; Kim, J. Computational Screening of Trillions of Metal-Organic Frameworks for High-Performance Methane Storage. ACS Appl. Mater. Interfaces 2021.

(4) Fang, Z.; Bueken, B.; De Vos, D. E.; Fischer, R. A. Defect-Engineered Metal-Organic Frameworks. Angew. Chem., Int. Ed. 2015, 54 (25), 7234-7254.

(5) Chong, S.; Thiele, G.; Kim, J. Excavating Hidden Adsorption Sites in Metal-Organic Frameworks Using Rational Defect Engineering. Nat. Commun. 2017, 8 (1), 1539.

(6) Boyd, P. G.; Chidambaram, A.; García-Díez, E.; Ireland, C. P.; Daff, T. D.; Bounds, R.; Gładysiak, A.; Schouwink, P.; Moosavi, S. M.; Maroto-Valer, M. M.; Reimer, J. A.; Navarro, J. A. R.; Woo, T. K.; Garcia, S.; Stylianou, K. C.; Smit, B. Data-Driven Design of Metal-Organic Frameworks for Wet Flue Gas $\mathrm{CO}_{2}$ Capture. Nature 2019, 576 (7786), 253-256.

(7) Rosen, A. S.; Notestein, J. M.; Snurr, R. Q. Structure-Activity Relationships That Identify Metal-Organic Framework Catalysts for Methane Activation. ACS Catal. 2019, 9 (4), 3576-3587.

(8) Teplensky, M. H.; Fantham, M.; Li, P.; Wang, T. C.; Mehta, J. P.; Young, L. J.; Moghadam, P. Z.; Hupp, J. T.; Farha, O. K.; Kaminski, C. F.; Fairen-Jimenez, D. Temperature Treatment of Highly Porous Zirconium-Containing Metal-Organic Frameworks Extends Drug Delivery Release. J. Am. Chem. Soc. 2017, 139 (22), $7522-$ 7532.

(9) Kim, H.; Yang, S.; Rao, S. R.; Narayanan, S.; Kapustin, E. A.; Furukawa, H.; Umans, A. S.; Yaghi, O. M.; Wang, E. N. Water Harvesting from Air with Metal-Organic Frameworks Powered by Natural Sunlight. Science 2017, 356 (6336), 430-434.

(10) Suh, B. L.; Chong, S.; Kim, J. Photochemically Induced Water Harvesting in Metal- 
Organic Framework. ACS Sustain. Chem. Eng. 2019, 7 (19), 15854-15859.

(11) Sun, L.; Campbell, M. G.; Dincă, M. Electrically Conductive Porous Metal-Organic Frameworks. Angew. Chem., Int. Ed. 2016, 55 (11), 3566-3579.

(12) Xie, L. S.; Skorupskii, G.; Dincă, M. Electrically Conductive Metal-Organic Frameworks. Chem. Rev. 2020, 120 (16), 8536-8580.

(13) Golomb, M. J.; Calbo, J.; Bristow, J. K.; Walsh, A. Ligand Engineering in Cu(II) Paddle Wheel Metal-Organic Frameworks for Enhanced Semiconductivity. J. Mater. Chem. A 2020, 8 (26), 13160-13165.

(14) Chong, S.; Kim, J. Rational Modifications of PCN-700 to Induce Electrical Conductivity: A Computational Study. Dalt. Trans. 2020, 49 (1), 102-113.

(15) Chong, S.; Park, D. T.; Kim, J. Exploring Guest-Dependent Photoconductivity in a Donor-Containing Metal-Organic Framework. J. Phys. Chem. C 2021, 125 (19), 10198-10206.

(16) Huang, X.; Sheng, P.; Tu, Z.; Zhang, F.; Wang, J.; Geng, H.; Zou, Y.; Di, C.; Yi, Y.; Sun, Y.; Xu, W.; Zhu, D. A Two-Dimensional $\pi-$ d Conjugated Coordination Polymer with Extremely High Electrical Conductivity and Ambipolar Transport Behaviour. Nat. Commun. 2015, 6 (1), 7408.

(17) Xie, L. S.; Sun, L.; Wan, R.; Park, S. S.; Degayner, J. A.; Hendon, C. H.; Dincă, M. Tunable Mixed-Valence Doping toward Record Electrical Conductivity in a ThreeDimensional Metal-Organic Framework. J. Am. Chem. Soc. 2018, 140 (24), 74117414.

(18) Song, X.; Wang, X.; Li, Y.; Zheng, C.; Zhang, B.; Di, C.; Li, F.; Jin, C.; Mi, W.; Chen, L.; Mi, W.; Chen, L; Hu, W. 2D Semiconducting Metal-Organic Framework Thin Films for Organic Spin Valves. Angew. Chem., Int. Ed. 2020, 132 (3), 1134-1139.

(19) Wu, G.; Huang, J.; Zang, Y.; He, J.; Xu, G. Porous Field-Effect Transistors Based on a Semiconductive Metal-Organic Framework. J. Am. Chem. Soc. 2017, 139 (4), 13601363.

(20) Sheberla, D.; Bachman, J. C.; Elias, J. S.; Sun, C. J.; Shao-Horn, Y.; Dincă, M. Conductive MOF Electrodes for Stable Supercapacitors with High Areal Capacitance. Nat. Mater. 2017, 16 (2), 220-224.

(21) Campbell, M. G.; Sheberla, D.; Liu, S. F.; Swager, T. M.; Dincă, M. Cu3 (Hexaiminotriphenylene) 2 : An Electrically Conductive 2D Metal-Organic Framework for Chemiresistive Sensing. Angew. Chem., Int. Ed. 2015, 54 (14), 4349-4352.

(22) Campbell, M. G.; Liu, S. F.; Swager, T. M.; Dincă, M. Chemiresistive Sensor Arrays from Conductive 2D Metal-Organic Frameworks. J. Am. Chem. Soc. 2015, 137 (43), 13780-13783.

(23) Meng, Z.; Aykanat, A.; Mirica, K. A. Welding Metallophthalocyanines into Bimetallic Molecular Meshes for Ultrasensitive, Low-Power Chemiresistive Detection of Gases. J. Am. Chem. Soc. 2019, 141 (5), 2046-2053.

(24) Miner, E. M.; Fukushima, T.; Sheberla, D.; Sun, L.; Surendranath, Y.; Dincă, M. Electrochemical Oxygen Reduction Catalysed by $\mathrm{Ni}_{3}(\text { Hexaiminotriphenylene) })_{2}$. Nat. 
Commun. 2016, 7 (1), 10942.

(25) Krause, S.; Hosono, N.; Kitagawa, S. Chemistry of Soft Porous Crystals: Structural Dynamics and Gas Adsorption Properties. Angew. Chem., Int. Ed. 2020, 59 (36), $15325-15341$.

(26) Coudert, F. X. Responsive Metal-Organic Frameworks and Framework Materials: Under Pressure, Taking the Heat, in the Spotlight, with Friends. Chem. Mater. 2015, 27 (6), 1905-1916.

(27) Vanduyfhuys, L.; Rogge, S. M. J.; Wieme, J.; Vandenbrande, S.; Maurin, G.; Waroquier, M.; Van Speybroeck, V. Thermodynamic Insight into Stimuli-Responsive Behaviour of Soft Porous Crystals. Nat. Commun. 2018, 9 (1), 204.

(28) Evans, J. D.; Bon, V.; Senkovska, I.; Lee, H.-C.; Kaskel, S. Four-Dimensional MetalOrganic Frameworks. Nat. Commun. 2020, 11 (1), 2690.

(29) Yanai, N.; Kitayama, K.; Hijikata, Y.; Sato, H.; Matsuda, R.; Kubota, Y.; Takata, M.; Mizuno, M.; Uemura, T.; Kitagawa, S. Gas Detection by Structural Variations of Fluorescent Guest Molecules in a Flexible Porous Coordination Polymer. Nat. Mater. 2011, 10 (10), 787-793.

(30) Qin, J. S.; Yuan, S.; Alsalme, A.; Zhou, H. C. Flexible Zirconium MOF as the Crystalline Sponge for Coordinative Alignment of Dicarboxylates. ACS Appl. Mater. Interfaces 2017, 9 (39), 33408-33412.

(31) Ling, S.; Slater, B. Unusually Large Band Gap Changes in Breathing Metal-Organic Framework Materials. J. Phys. Chem. C 2015, 119 (29), 16667-16677.

(32) Choi, H. J.; Dincă, M.; Long, J. R. Broadly Hysteretic $\mathrm{H}_{2}$ Adsorption in the Microporous Metal-Organic Framework Co(1,4-Benzenedipyrazolate). J. Am. Chem. Soc. 2008, 130 (25), 7848-7850.

(33) Galli, S.; Masciocchi, N.; Colombo, V.; Maspero, A.; Palmisano, G.; López-Garzón, F. J.; Domingo-García, M.; Fernández-Morales, I.; Barea, E.; Navarro, J. A. R. Adsorption of Harmful Organic Vapors by Flexible Hydrophobic Bis-Pyrazolate Based MOFs. Chem. Mater. 2010, 22 (5), 1664-1672.

(34) Mason, J. A.; Oktawiec, J.; Taylor, M. K.; Hudson, M. R.; Rodriguez, J.; Bachman, J. E.; Gonzalez, M. I.; Cervellino, A.; Guagliardi, A.; Brown, C. M.; Llewellyn, P. L.; Masciocchi, N.; Long, J. R. Methane Storage in Flexible Metal-Organic Frameworks with Intrinsic Thermal Management. Nature 2015, 527 (7578), 357-361.

(35) Barthelet, K.; Marrot, J.; Riou, D.; Férey, G. A Breathing Hybrid Organic-Inorganic Solid with Very Large Pores and High Magnetic Characteristics. Angew. Chem., Int. Ed. 2002, 41 (2), 281.

(36) Loiseau, T.; Serre, C.; Huguenard, C.; Fink, G.; Taulelle, F.; Henry, M.; Bataille, T.; Férey, G. A Rationale for the Large Breathing of the Porous Aluminum Terephthalate (MIL-53) Upon Hydration. Chem. - Eur. J. 2004, 10 (6), 1373-1382.

(37) Wentz, H. C.; Skorupskii, G.; Bonfim, A. B.; Mancuso, J. L.; Hendon, C. H.; Oriel, E. H.; Sazama, G. T.; Campbell, M. G. Switchable Electrical Conductivity in a ThreeDimensional Metal-Organic Framework via Reversible Ligand n-Doping. Chem. Sci. 2020, 11 (5), 1342-1346. 
(38) Garai, B.; Mallick, A.; Banerjee, R. Photochromic Metal-Organic Frameworks for Inkless and Erasable Printing. Chem. Sci. 2016, 7 (3), 2195-2200.

(39) Xie, Y.-X.; Zhao, W.-N.; Li, G.-C.; Liu, P.-F.; Han, L. A Naphthalenediimide-Based Metal-Organic Framework and Thin Film Exhibiting Photochromic and Electrochromic Properties. Inorg. Chem. 2016, 55 (2), 549-551.

(40) Guo, Z.; Panda, D. K.; Maity, K.; Lindsey, D.; Parker, T. G.; Albrecht-Schmitt, T. E.; Barreda-Esparza, J. L.; Xiong, P.; Zhou, W.; Saha, S. Modulating the Electrical Conductivity of Metal-Organic Framework Films with Intercalated Guest $\pi$-Systems. J. Mater. Chem. C 2016, 4 (5), 894-899.

(41) Guo, Z.; Panda, D. K.; Gordillo, M. A.; Khatun, A.; Wu, H.; Zhou, W.; Saha, S. Lowering Band Gap of an Electroactive Metal-Organic Framework via Complementary Guest Intercalation. ACS Appl. Mater. Interfaces 2017, 9 (38), 3241332417.

(42) Castaldelli, E.; Imalka Jayawardena, K. D. G.; Cox, D. C.; Clarkson, G. J.; Walton, R. I.; Le-Quang, L.; Chauvin, J.; Silva, S. R. P.; Demets, G. J. F. Electrical Semiconduction Modulated by Light in a Cobalt and Naphthalene Diimide MetalOrganic Framework. Nat. Commun. 2017, 8 (1).

(43) Zhou, W.; Yildirim, T. Lattice Dynamics of Metal-Organic Frameworks: Neutron Inelastic Scattering and First-Principles Calculations. Phys. Rev. B 2006, 74 (18), 180301.

(44) Rogge, S. M. J.; Wieme, J.; Vanduyfhuys, L.; Vandenbrande, S.; Maurin, G.; Verstraelen, T.; Waroquier, M.; Van Speybroeck, V. Thermodynamic Insight in the High-Pressure Behavior of UiO-66: Effect of Linker Defects and Linker Expansion. Chem. Mater. 2016, 28 (16), 5721-5732.

(45) Boyd, P. G.; Moosavi, S. M.; Witman, M.; Smit, B. Force-Field Prediction of Materials Properties in Metal-Organic Frameworks. J. Phys. Chem. Lett. 2017, 8 (2), 357-363.

(46) Rogge, S. M. J.; Vanduyfhuys, L.; Ghysels, A.; Waroquier, M.; Verstraelen, T.; Maurin, G.; Van Speybroeck, V. A Comparison of Barostats for the Mechanical Characterization of Metal-Organic Frameworks. J. Chem. Theory Comput. 2015, 11 (12), 5583-5597.

(47) Rogge, S. M. J.; Waroquier, M.; Van Speybroeck, V. Reliably Modeling the Mechanical Stability of Rigid and Flexible Metal-Organic Frameworks. Acc. Chem. Res. 2018, 51 (1), 138-148.

(48) Wieme, J.; Vanduyfhuys, L.; Rogge, S. M. J.; Waroquier, M.; Van Speybroeck, V. Exploring the Flexibility of MIL-47(V)-Type Materials Using Force Field Molecular Dynamics Simulations. J. Phys. Chem. C 2016, 120 (27), 14934-14947.

(49) Hoffmann, R. How Chemistry and Physics Meet in the Solid State. Angew. Chem., Int. Ed. 1987, 26 (9), 846-878.

(50) Foster, M. E.; Sohlberg, K.; Spataru, C. D.; Allendorf, M. D. Proposed Modification of the Graphene Analogue $\mathrm{Ni}_{3}(\mathrm{HITP})_{2}$ To Yield a Semiconducting Material. J. Phys. Chem. C 2016, 120 (27), 15001-15008. 
(51) Dong, R.; Han, P.; Arora, H.; Ballabio, M.; Karakus, M.; Zhang, Z.; Shekhar, C.; Adler, P.; Petkov, P. S.; Erbe, A.; Mannsfeld, S. C. B.; Felser, C.; Heine, T.; Bonn, M.; Feng, X.; Cánovas, E. High-Mobility Band-like Charge Transport in a Semiconducting Two-Dimensional Metal-Organic Framework. Nat. Mater. 2018, 17 (11), 1027-1032.

(52) Chen, L.; Mowat, J. P. S.; Fairen-Jimenez, D.; Morrison, C. A.; Thompson, S. P.; Wright, P. A.; Düren, T. Elucidating the Breathing of the Metal-Organic Framework MIL-53(Sc) with Ab Initio Molecular Dynamics Simulations and in Situ X-Ray Powder Diffraction Experiments. J. Am. Chem. Soc. 2013, 135 (42), 15763-15773.

(53) Reinsch, H.; Pillai, R. S.; Siegel, R.; Senker, J.; Lieb, A.; Maurin, G.; Stock, N. Structure and Properties of Al-MIL-53-ADP, a Breathing MOF Based on the Aliphatic Linker Molecule Adipic Acid. Dalt. Trans. 2016, 45 (10), 4179-4186.

(54) Wieme, J.; Rogge, S. M. J.; Yot, P. G.; Vanduyfhuys, L.; Lee, S. K.; Chang, J. S.; Waroquier, M.; Maurin, G.; Van Speybroeck, V. Pillared-Layered Metal-Organic Frameworks for Mechanical Energy Storage Applications. J. Mater. Chem. A 2019, 7 (39), 22663-22674.

(55) Yang, X.; Si, L.; Xie, G.; Zhang, L.; Guo, D.; Luo, J. Intrinsic Adsorption Behaviour Related to the Structural and Mechanical Properties of Flexible Metal-Organic Frameworks Co(Bdp). Comput. Mater. Sci. 2020, 177 (39), 109543.

(56) Kresse, G.; Hafner, J. Ab Initio Molecular Dynamics for Liquid Metals. Phys. Rev. B 1993, 47 (1), 558-561.

(57) Kresse, G.; Furthmüller, J. Efficiency of Ab-Initio Total Energy Calculations for Metals and Semiconductors Using a Plane-Wave Basis Set. Comput. Mater. Sci. 1996, $6(1), 15-50$.

(58) Kresse, G.; Furthmüller, J. Efficient Iterative Schemes for Ab Initio Total-Energy Calculations Using a Plane-Wave Basis Set. Phys. Rev. B 1996, 54 (16), 11169-11186.

(59) Blöchl, P. E. Projector Augmented-Wave Method. Phys. Rev. B 1994, 50 (24), 1795317979.

(60) Kresse, G.; Joubert, D. From Ultrasoft Pseudopotentials to the Projector AugmentedWave Method. Phys. Rev. B 1999, 59 (3), 1758-1775.

(61) Perdew, J. P.; Burke, K.; Ernzerhof, M. Generalized Gradient Approximation Made Simple. Phys. Rev. Lett. 1996, 77 (18), 3865-3868.

(62) Grimme, S.; Ehrlich, S.; Goerigk, L. Effect of the Damping Function in Dispersion Corrected Density Functional Theory. J. Comput. Chem. 2011, 32 (7), 1456-1465.

(63) Krukau, A. V.; Vydrov, O. A.; Izmaylov, A. F.; Scuseria, G. E. Influence of the Exchange Screening Parameter on the Performance of Screened Hybrid Functionals. $J$. Chem. Phys. 2006, 125 (22), 224106.

(64) Moellmann, J.; Grimme, S. DFT-D3 Study of Some Molecular Crystals. J. Phys. Chem. C 2014, 118 (14), 7615-7621.

(65) M Ganose, A.; J Jackson, A.; O Scanlon, D. Sumo: Command-Line Tools for Plotting and Analysis of Periodic Ab Initio Calculations. J. Open Source Softw. 2018, 3 (28), 717. 
(66) Vanduyfhuys, L.; Vandenbrande, S.; Verstraelen, T.; Schmid, R.; Waroquier, M.; Van Speybroeck, V. QuickFF: A Program for a Quick and Easy Derivation of Force Fields for Metal-Organic Frameworks from Ab initio Input. J. Comput. Chem. 2015, 36 (13), $1015-1027$.

(67) Vanduyfhuys, L.; Vandenbrande, S.; Wieme, J.; Waroquier, M.; Verstraelen, T.; Van Speybroeck, V. Extension of the QuickFF Force Field Protocol for an Improved Accuracy of Structural, Vibrational, Mechanical and Thermal Properties of MetalOrganic Frameworks. J. Comput. Chem. 2018, 39 (16), 999-1011.

(68) Verstraelen, T.; Vandenbrande, S.; Heidar-Zadeh, F.; Vanduyfhuys, L.; Van Speybroeck, V.; Waroquier, M.; Ayers, P. W. Minimal Basis Iterative Stockholder: Atoms in Molecules for Force-Field Development. J. Chem. Theory Comput. 2016, 12 (8), 3894-3912.

(69) Mortensen, J. J.; Hansen, L. B.; Jacobsen, K. W. Real-Space Grid Implementation of the Projector Augmented Wave Method. Phys. Rev. B 2005, 71 (3), 035109.

(70) Enkovaara, J.; Rostgaard, C.; Mortensen, J. J.; Chen, J.; Dułak, M.; Ferrighi, L.; Gavnholt, J.; Glinsvad, C.; Haikola, V.; Hansen, H. A.; Kristoffersen, H. H.; Kuisma, M.; Larsen, A. H.; Lehtovaara, L.; Ljungberg, M.; Lopez-Acevedo, O.; Moses, P. G.; Ojanen, J.; Olsen, T.; Petzold, V.; Romero, N. A.; Stausholm-Møller, J.; Strange, M.; Tritsaris, G. A.; Vanin, M.; Walter, M.; Hammer, B.; Häkkinen, H.; Madsen, G. K. H.; Nieminen, R. M.; Nørskov, J. K.; Puska, M.; Rantala, T. T.; Schiøtz, J.; Thygesen, K. S.; Jacobsen, K. W. Electronic Structure Calculations with GPAW: A Real-Space Implementation of the Projector Augmented-Wave Method. J. Phys. Condens. Matter 2010, 22 (25), 253202.

(71) Chen, J.; Martínez, T. J. QTPIE: Charge Transfer with Polarization Current Equalization. A Fluctuating Charge Model with Correct Asymptotics. Chem. Phys. Lett. 2007, 438 (4-6), 315-320.

(72) Lii, J. H.; Allinger, N. L. Molecular Mechanics. The MM3 Force Field for Hydrocarbons. 2. Vibrational Frequencies and Thermodynamics. J. Am. Chem. Soc. 1989, 111 (23), 8566-8575.

(73) Verstraelen, T.; Vanduyfhuys, L.; Vandenbrande, S.; Rogge, S. M. J. Yaff, yet Another Force Field. https://molmod.ugent.be/software/

(74) Nosé, S. A Unified Formulation of the Constant Temperature Molecular Dynamics Methods. J. Chem. Phys. 1984, 81 (1), 511-519.

(75) Hoover, W. G. Canonical Dynamics: Equilibrium Phase-Space Distributions. Phys. Rev. A 1985, 31 (3), 1695-1697.

(76) Martyna, G. J.; Tuckerman, M. E.; Tobias, D. J.; Klein, M. L. Explicit Reversible Integrators for Extended Systems Dynamics. Mol. Phys. 1996, 87 (5), 1117-1157.

(77) Savitzky, A.; Golay, M. J. E. Smoothing and Differentiation of Data by Simplified Least Squares Procedures. Anal. Chem. 1964, 36 (8), 1627-1639. 


\section{Flexible \& conductive MOFs}

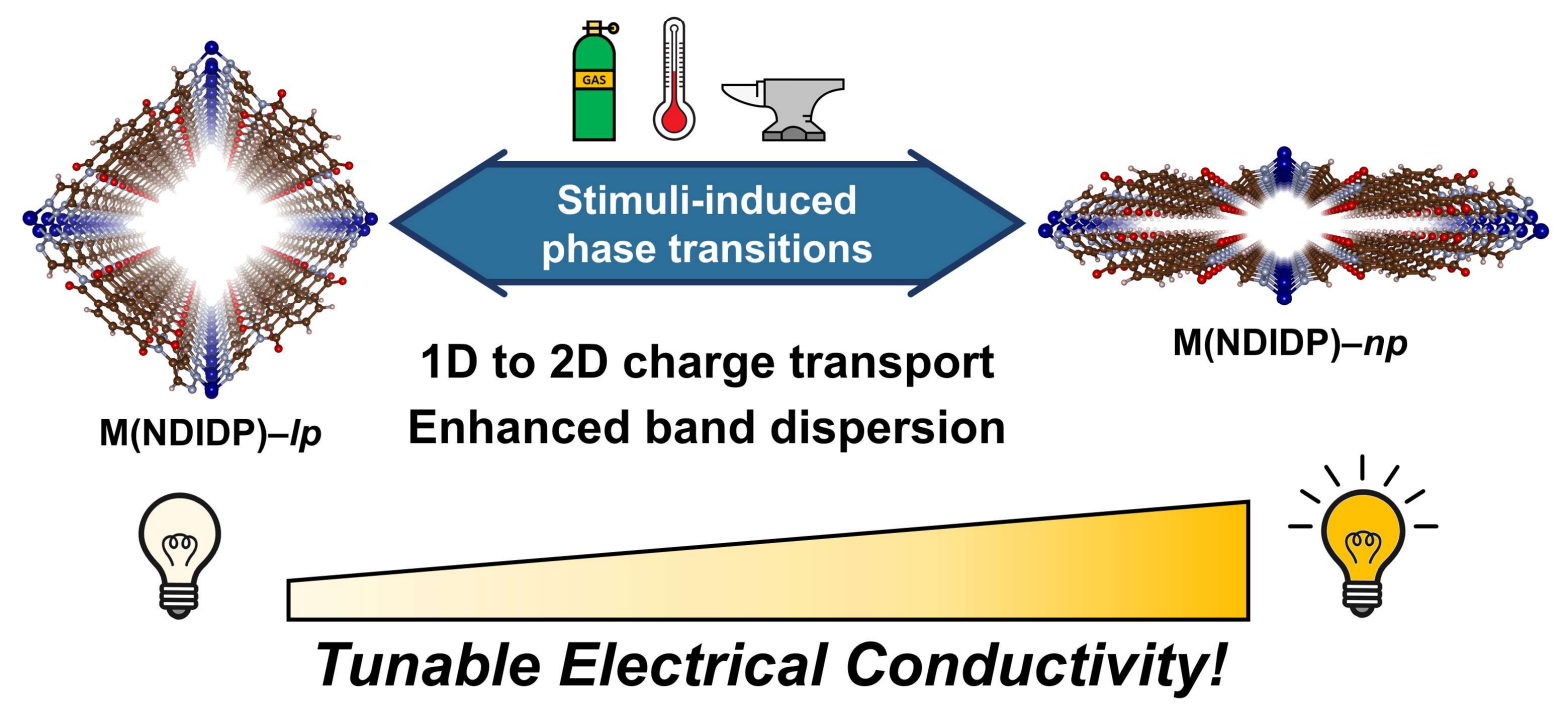

